\title{
Classificação Estrutural e Qualidade da Madeira do Clone GT 1 de Hevea brasiliensis Muell. Arg.
}

\author{
Elaine Cristina Leonello ${ }^{1}$, Adriano Wagner Ballarin², Jéssica Monari Ohto ${ }^{3}$, \\ Hernando Alfonso Lara Palma ${ }^{2}$, Javier Farago Escobar ${ }^{1}$ \\ ${ }^{1}$ Programa de Pós-graduação em Ciência Florestal, Faculdade de Ciências Agronômicas - FCA, \\ Universidade Estadual Paulista - UNESP \\ ${ }^{2}$ Faculdade de Ciências Agronômicas - FCA, Universidade Estadual Paulista - UNESP \\ ${ }^{3}$ Curso de Engenharia Florestal, Faculdade de Ciências Agronômicas - FCA, Universidade Estadual Paulista - UNESP
}

\begin{abstract}
RESUMO
O látex é o principal produto extraído das árvores de seringueira (Hevea brasiliensis). No Brasil, ao final do ciclo produtivo de látex, a madeira dessas árvores é utilizada tradicionalmente para fins energéticos, mas diversos trabalhos internacionais relatam práticas consolidadas de agregação de valor a ela. O objetivo deste trabalho foi avaliar a qualidade da madeira e classificá-la estruturalmente a partir de propriedades mecânicas do lenho de árvores do clone comercial GT 1 de seringueira. Foram utilizadas seis árvores provenientes de plantio de 20 anos de idade da cidade de Itajobi-SP. Da tora inferior (desde a base até a altura do DAP), foram produzidos corpos de prova de dimensões reduzidas, na direção radial do lenho, para avaliação da qualidade pelos ensaios de compressão paralela às fibras, flexão estática e dureza Janka; da tora superior (do DAP até a altura de 2,50 m), foram produzidos dois corpos de prova por árvore, para a classificação estrutural baseada no ensaio de compressão paralela às fibras (NBR 7190, 1997 (ABNT, 1997)). A madeira foi classificada estruturalmente como classe C40. Os resultados revelaram que a resistência não apresentou diferença estatisticamente significativa na direção radial (a menos da dureza Janka), sendo observada, no entanto, tendência de aumento no sentido medula-casca.
\end{abstract}

Palavras-chave: propriedades mecânicas, tecnologia, seringueira.

\section{Quality of Wood and Structural Classification of Hevea brasiliensis Clone GT 1}

\begin{abstract}
Latex is the main product extracted from rubber trees (Hevea brasiliensis). In Brazil, at the end of the production cycle of latex, the wood of rubber tree is traditionally used for energy purposes, but several international studies have reported consolidated practices of adding value to it. The objective of this paper was to evaluate the quality of wood and classify it structurally based on its mechanical properties. Six 20-year-old trees of the clone GT 1 of rubber tree proceeding from Itajobi, State of Sao Paulo, Brazil were sampled. Reduced dimensions specimens in the radial direction of the wood were produced to evaluate the quality by compression parallel to the grain, static bending and Janka hardness tests. Two specimens, one from the lower log (since the ground up to breast height) and one from the higher log (from breast height up to $2.50 \mathrm{~m}$ ) were produced for structural classification of the wood based on the characteristic strength in compression parallel to the grain (NBR 7190 norm, 1997). The wood was classified as C40 ( $f_{\text {cok }} \geq 40 \mathrm{MPa}$ ) class. Results revealed that the strength was not statistically different in the radial direction (except for the Janka hardness), though tending to increase from pith to bark.
\end{abstract}

Keywords: wood mechanical properties, technology, rubber tree. 


\section{INTRODUÇÃO}

Apesar de ser originária da região Amazônica, a Hevea brasiliensis tem a maior área plantada (cerca de $90 \%$ ) no continente asiático (Zhao, 2008). Isso se deve, sobretudo, a fatores climáticos e fitopatológicos: semelhança climática com a região de origem e ausência do fungo Mycrocyclus ulei, causador da doença "mal das folhas". A importância dos plantios de Hevea brasiliensis é associada ao látex, que tem grande valor comercial, por ser a principal matériaprima na fabricação da borracha natural (IAC, 2009).

A madeira de seringueira, apesar de possuir potencial tecnológico com grande leque de aplicações, no Brasil é utilizada com muita frequência em aplicações de menor valor agregado, como lenha e carvão. Essa prática se estende a muitos outros países, que utilizam a madeira para fins energéticos e até residuais (Colloquium on rubberwood: resources and technologies, 1998; May \& Gonçalves, 1999; Balsiger et al., 2000; Lorenzi, 2000; Teixeira et al., 2001; IAPAR, 2004; Okino et al., 2004).

A avaliação do potencial tecnológico de espécies florestais com disponibilidade comercial é iniciada pela determinação de suas propriedades físico-mecânicas (Ballarin, 2000 apud Benjamin, 2006), sendo que as propriedades mecânicas avaliam a aptidão da madeira em suportar as solicitações mecânicas. Essas são avaliadas em duas direções principais, paralela e normal às fibras, e são normalmente mensuradas considerandose a resistência e a rigidez da madeira. Dentre as propriedades mecânicas mensuradas na madeira, a compressão paralela às fibras é, no enfoque nacional, a propriedade de maior importância. Ela tem boa correlação com quase todas as demais propriedades mecânicas da madeira e é o parâmetro balizador na classificação estrutural desse material.

Santana et al. (2001), em estudo da madeira de quatro clones de Hevea brasiliensis, observaram resistência à compressão paralela às fibras variando de 41,7 a 43,3 MPa. Matan \& Kyokong (2003) relataram valores de $39 \mathrm{MPa}$ em árvores de 25 a 30 anos de idade na Tailândia e Hong (1996) cita que Lee et al. (1965) encontraram o valor de 32,2 MPa para a mesma propriedade. No Brasil, Eufrade Junior et al. (2011) reportaram que a madeira de árvores de 29 anos do clone RRIM 600 de seringueira apresentou resistência média à compressão paralela às fibras de 43,53 MPa, com coeficiente de variação de 8,61\% e com resistência característica de 41,90 $\mathrm{MPa}$, enquadrando-a na classe de resistência C40.

O ensaio de flexão estática é especificado na série normal de caracterização de madeiras pela grande maioria dos códigos normativos internacionais. Apesar de se constituir em um ensaio de determinação "impura" da resistência da madeira, apresenta como vantagem a facilidade de execução e seus fundamentos são empregados nas máquinas de classificação MSR (Machine Stress Rating). Santana et al. (2001), no estudo já relatado, reportaram módulo de ruptura à flexão (MOR) variando de 81,5 a $96,9 \mathrm{MPa}$ para a madeira de seringueira. Lee et al. (1979 apud Lim et al., 2003) encontraram os valores de 66 e 9.240 MPa para MOR e MOE (módulo de elasticidade à flexão estática), respectivamente.

$\mathrm{Na}$ avaliação de madeiras para diversos usos, a dureza Janka evidencia-se, também, como propriedade de interesse e fácil mensuração em condições de campo. Embora, para alguns autores, seja considerada uma propriedade mecânica sem aplicação imediata ou direta dos seus resultados, subsiste como propriedade de real importância na caracterização mecânica de madeiras por ser importante parâmetro de comparação entre espécies (Rocha, 2003). Valores encontrados por Santana et al. (2001) para a dureza Janka (12\% de umidade) da madeira de seringueira, na direção paralela às fibras, variam entre 2.120 e $5.370 \mathrm{~N}$, já Lee et al. (1979 apud Lim et al., 2003) encontraram valor de dureza para madeira de seringueira úmida de $3.030 \mathrm{~N}$ e para a madeira seca ao ar de $4.320 \mathrm{~N}$.

Quanto à variação radial das propriedades analisadas, não há trabalhos na literatura que permitam fazer avaliações específicas para esta espécie; no geral, a tendência da densidade aparente e das propriedades mecânicas da madeira é apresentar aumento no sentido medula-casca (Panshin \& Zeeuw, 1980; Tomazello Filho, 1985, 1987; Bodig \& Jayne, 1993).

Este trabalho teve o objetivo de classificar estruturalmente a madeira de árvores do clone GT 1 de seringueira de árvores de 20 anos de idade a 
partir da resistência à compressão paralela às fibras e avaliar sua qualidade, por meio da variação radial das propriedades mecânicas de compressão paralela às fibras, flexão estática e dureza Janka.

\section{MATERIAL E MÉTODOS}

As árvores do clone GT 1, de 20 anos de idade, foram retiradas de plantação na cidade de Itajobi, SP, localizada nas coordenadas geográficas $21^{\circ} 18^{\prime} \mathrm{S}$ e $49^{\circ}$ 01' O. Foram selecionadas seis árvores representantes do extrato superior das classes diamétricas da população, visando à possibilidade de se retirar a maior quantidade de corpos de prova na direção radial do tronco.

De cada árvore foi retirada a partir da base uma tora de 2,5 m de comprimento. Da parte inferior da tora (desde a base até o DAP) foi desdobrado um pranchão de $80 \mathrm{~mm}$ de espessura, cuidando-se para que ele contivesse a medula. Do topo do pranchão, foi retirada uma faixa diametral de $70 \mathrm{~mm}$ de espessura. Do pranchão restante, foram descartados $30 \mathrm{~mm}$ de madeira, centralizados na medula, para que ela fosse completamente eliminada. Posteriormente foram serrados caibros com seção transversal de $(35 \times 35) \mathrm{mm}^{2}$, ao longo dos dois raios do pranchão, partindo-se da região mais interna (próxima à medula) em direção às extremidades. Dessa forma, manteve-se o paralelismo dos caibros em relação ao eixo medular da árvore.

Da parte superior da tora (desde 1,3 $\mathrm{m}$ de altura até o topo da tora) foram retirados dois corpos de prova por árvore para o ensaio de compressão paralela às fibras, com sobremedida em relação às dimensões normativas, totalizando 12 corpos de prova para a caracterização simplificada da resistência da madeira, conforme sugere a norma NBR 7190 (ABNT, 1997).

As faixas diametrais, os caibros e os corpos de prova de compressão foram levados para câmara de climatização, onde se mantiveram em condições de umidade relativa do ar de $65 \%$ e temperatura de $20{ }^{\circ} \mathrm{C}$, até atingir umidade de equilíbrio próxima de $12 \%$, de tal forma que deles, com exceção da faixa diametral, pudessem ser desdobrados os corpos de prova, em suas dimensões finais, sem que sofressem retrações adicionais significativas.
Os corpos de prova de compressão paralela às fibras, destinados à caracterização simplificada da resistência da madeira, de dimensões nominais de $50 \times 50 \times 150 \mathrm{~mm}$ foram ensaiados segundo a NBR 7190 (ABNT, 1997), conforme ilustra a Figura 1. Depois da avaliação da propriedade pretendida (resistência: $f_{c 0}$ ), os resultados foram corrigidos para a umidade de referência de $12 \%$, a partir da expressão sugerida pelo código normativo.

Utilizando-se de prerrogativas da NBR 7190 (ABNT, 1997), que permitem a redução das dimensões dos corpos de prova para até $18 \mathrm{~mm}$ de lado, desde que mantidas as proporcionalidades dimensionais originais entre os lados e entre os lados e os comprimentos (1:3 - no ensaio de compressão paralela às fibras; e 1:23 - no ensaio de flexão estática), os corpos de prova destinados à avaliação da qualidade da madeira através da variação radial dos parâmetros mecânicos observados tiveram suas dimensões nominais da seção transversal reduzidas para um módulo de $20 \mathrm{~mm}$ (ao invés dos $50 \mathrm{~mm}$, utilizados correntemente) sem prejuízo na qualidade dos resultados. Portanto, como ilustrado na Figura 2, os corpos de prova "A", de dimensões nominais $20 \times 20 \times 60 \mathrm{~mm}$, foram destinados à compressão paralela às fibras para determinação de resistência, e os corpos de prova "B", de dimensões nominais de $20 \times 20 \times 460 \mathrm{~mm}$, foram destinados ao ensaio de flexão estática, para determinação de resistência e rigidez.

Os ensaios de dureza Janka foram executados exclusivamente na direção longitudinal segundo

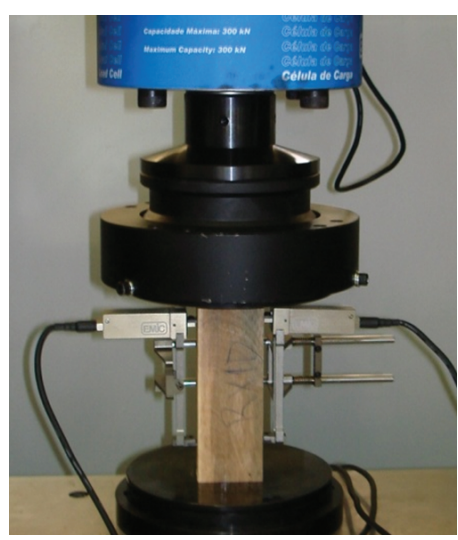

Figura 1. Ensaio de compressão paralela às fibras em andamento.

Figure 1. Compression parallel to grain test in progress. 
a NBR 7190 (ABNT, 1997) a menos da dimensão dos corpos de prova, que foram substituídos pelas faixas diametrais obtidas das árvores amostradas. Para correspondência dos ensaios mecânicos na direção radial, os ensaios de dureza foram realizados nas posições que distam 32,5, 71,5 e 110,5 mm da medula em cada raio (distâncias que correspondem à posição central dos caibros desdobrados do pranchão central). A Figura 3 ilustra uma faixa diametral com os pontos marcados para realização do ensaio de dureza Janka.

Todos os ensaios foram realizados no Laboratório de Ensaio de materiais da Faculdade de Ciências Agronômicas - UNESP - Botucatu, com uso de uma máquina universal de ensaios EMIC-DL-30000, servocontrolada, com capacidade de carga de $300 \mathrm{kN}$.

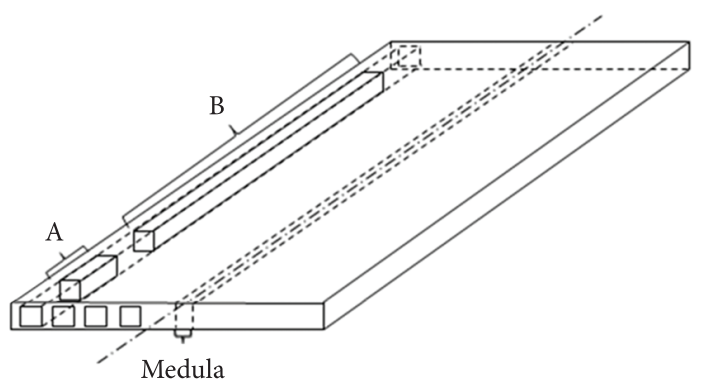

Figura 2. Esquema de obtenção dos corpos de prova para os ensaios mecânicos de avaliação da qualidade da madeira do clone GT 1 de seringueira.

Figure 2. Scheme for obtaining specimens of mechanical tests - wood quality evaluation of clone GT 1 of rubber trees.

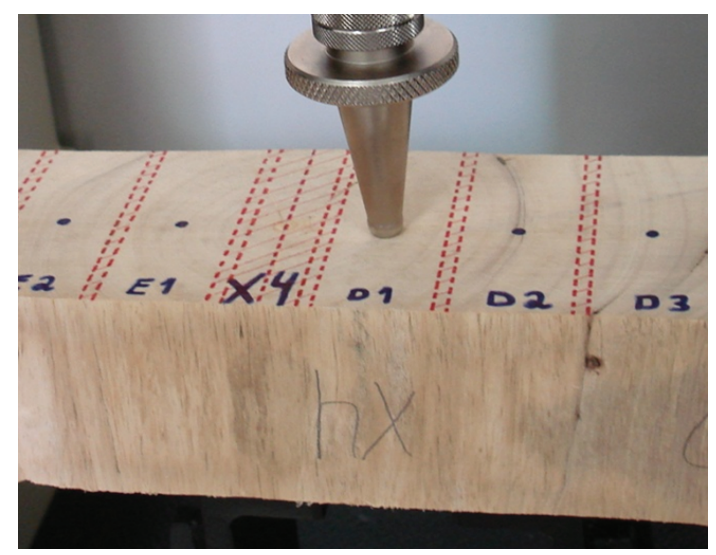

Figura 3. Ensaio de dureza Janka em andamento. Figure 3. Janka hardness test in progress.
As médias dos resultados na direção radial foram comparadas, pelo teste de Tukey ( $\mathrm{p}>0,05)$, para as propriedades mecânicas utilizadas na avaliação da qualidade da madeira de árvores do clone GT 1.

A tipologia de ruptura dos corpos de prova foi caracterizada com auxílio da norma americana ASTM D143 (ASTM, 2007) por falta de especificação normativa nacional sobre o assunto.

\section{RESULTADOS E DISCUSSÃO}

\subsection{Caracterização simplificada da resistência da madeira}

O valor médio da resistência à compressão paralela às fibras a $12 \%$ de umidade foi $49,83 \mathrm{MPa}$, com coeficiente de variação dos resultados de 7,49\%. Este valor médio é superior aos encontrados por Santana et al. (2001) para outros quatro clones da mesma espécie (entre 41,7 e 43,3 $\mathrm{MPa}$ ) e por Silva (2010), que obteve resistência à compressão paralela às fibras média de 41,87 $\mathrm{MPa}$, com coeficiente de variação de $13 \%$ na condição de $12 \%$ de umidade. A resistência à compressão paralela às fibras obtida foi também superior à representada por Eufrade Junior et al. (2011) para a madeira de árvores de 29 anos do clone RRIM 600, de 43,53 MPa.

O valor característico da resistência da madeira obtida neste ensaio foi de 49,63 MPa, o que permite que a madeira do clone GT 1 de seringueira possa ser classificada como de classe de resistência 40 (C40), da mesma forma que o constatado por Eufrade Junior et al. (2011) para o clone RRIM 600.

A Figura 4 apresenta os resultados de resistência de cada corpo de prova corrigido para a umidade padrão de $12 \%$ e indica o valor característico calculado para a resistência. Evidencia-se na figura a homogeneidade do lote estudado, o que resultou num valor característico muito próximo do valor médio.

Dentre as caracterizações de ruptura encontradas, o esmagamento (crushing) representou mais de $90 \%$ do lote, indicando a homogeneidade e o padrão normal de ruptura dos corpos de prova no ensaio. 


\subsection{Avaliação radial da qualidade da madeira}

Os resultados das propriedades mecânicas (resistência à compressão paralela às fibras - $\mathrm{f}_{c 0}$, resistência $-\mathrm{f}_{M}-\mathrm{e}$ rigidez $-\mathrm{E}_{\mathrm{M}}-$ no ensaio de flexão estática e dureza Janka $-\mathrm{f}_{\mathrm{H} 0}$ ) para avaliação da qualidade da madeira do clone GT 1 de seringueira na direção radial estão apresentados na Tabela 1 juntamente com o teste de comparação de médias (Tukey - 5\% de significância).

A madeira do clone GT 1 teve resistência à compressão paralela às fibras variando radialmente entre 42,77 e 43,88 $\mathrm{MPa}$, com coeficiente de cada posição radial estudada variando de 6,51 a 10,31\%. $\mathrm{O}$ teste estatístico de análise de variação

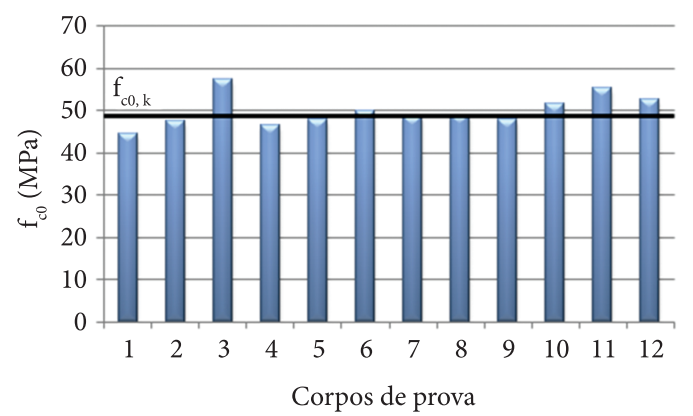

Figura 4. Resistência da madeira à compressão paralela às fibras $\left(\mathrm{f}_{\mathrm{c} 0}\right)$ corrigida para $12 \%$ de umidade com indicação do valor característico $\left(\mathrm{f}_{\mathrm{c} 0 \mathrm{k}}\right)$.

Figure 4. Compression parallel to grain strength $\left(f_{c 0}\right)$ reported to $12 \%$ MC with an indication of the characteristic value $\left(\mathrm{f}_{\mathrm{c} 0, \mathrm{k}}\right)$.
( $p$ valor $=0,749)$ revelou não haver diferença radial significativa para esta propriedade.

A ruptura de $94,5 \%$ dos corpos de prova ensaiados à compressão paralela às fibras foi enquadrada no tipo esmagamento (crushing), mostrando a homogeneidade e o padrão normal de ruptura dos corpos de prova. Somente 3,3\% dos corpos de prova apresentaram rupturas dos tipos compressão e cisalhamento paralelo à grã (compression and shearing parallel to grain) $\mathrm{e}$ "rolamento" na extremidade (brooming or end rolling) que são rupturas consideradas frágeis. Esses resultados foram descartados da base de dados do trabalho, atendendo a indicações da ASTM D143-94 (ASTM, 2007).

A resistência à flexão estática variou de 82,49 a 84,69 MPa e a rigidez variou de 9.877 a $10.107 \mathrm{MPa}$. Os coeficientes de variação encontrados foram de 11,61 a 16,52\% para resistência e de 9,12 e $16,22 \%$ para rigidez, respectivamente. Radialmente, a análise de variância ( $\mathrm{p}$ valor de 0,911 e 0,891 para resistência e rigidez, respectivamente) revelou novamente não haver diferença estatística de resultados entre as distâncias radiais consideradas. Apesar de não ser estatisticamente significante a variação radial da resistência e da rigidez no ensaio de flexão, houve a tendência de aumento no sentido medula-casca de ambas as propriedades em questão, revelando discreta influência da juvenilidade da madeira.

Os valores de resistência à flexão reportados para outros clones de Hevea brasiliensis por

Tabela 1. Variação radial das propriedades da madeira de árvores do clone GT 1 de seringueira.

Table 1. Radial variation of the mechanical properties of wood of clone GT1 of rubber trees.

\begin{tabular}{|c|c|c|c|c|c|}
\hline \multirow{2}{*}{\multicolumn{2}{|c|}{$\begin{array}{c}\text { Propriedades } \\
\text { mecânicas }\end{array}$}} & \multicolumn{3}{|c|}{ Posição radial (mm) } & \multirow{2}{*}{$\mathbf{p}$ valor } \\
\hline & & 32,5 & 71,5 & 110,5 & \\
\hline \multirow{2}{*}{$\mathbf{f}_{\mathrm{c} 0}$} & Média (MPa) & $43,88^{\mathrm{a}}$ & $42,77^{\mathrm{a}}$ & $43,81^{\mathrm{a}}$ & \multirow{2}{*}{0,749} \\
\hline & CV (\%) & 6,51 & 10,31 & 9,84 & \\
\hline \multirow{2}{*}{$\mathbf{f}_{\mathrm{M}}$} & Média (MPa) & $82,49^{\mathrm{a}}$ & $83,90^{\mathrm{a}}$ & $84,69^{\mathrm{a}}$ & \multirow{2}{*}{0,911} \\
\hline & CV (\%) & 14,17 & 11,61 & 16,52 & \\
\hline \multirow{2}{*}{$\mathrm{E}_{\mathrm{M}}$} & Média (MPa) & $9,926^{\mathrm{a}}$ & $10,107^{\mathrm{a}}$ & $9,877^{\mathrm{a}}$ & \multirow{2}{*}{0,891} \\
\hline & CV (\%) & 9,12 & 10,34 & 16,22 & \\
\hline \multirow{2}{*}{$\mathbf{f}_{\mathrm{H} 0}$} & Média (MPa) & $57,28^{\mathrm{b}}$ & $63,06^{\mathrm{ab}}$ & $67,06^{\mathrm{a}}$ & \multirow{2}{*}{0,022} \\
\hline & CV (\%) & 11,18 & 14,84 & 8,03 & \\
\hline
\end{tabular}

Em uma mesma linha, valores seguidos de ao menos uma letra igual não diferem estatisticamente (Tukey; $p>0,05$ ). $\mathrm{f}_{\mathrm{co}}$ - resistência à compressão paralela às fibras; $\mathrm{f}_{\mathrm{M}}$ - resistência à flexão; $\mathrm{E}_{\mathrm{M}}$ - rigidez à flexão; $\mathrm{f}_{\mathrm{H} 0}$ - dureza Janka paralela às fibras. 
Santana et al. (2001) foram de 81,5 a 96,9 $\mathrm{MPa}$, próximos aos obtidos neste trabalho. Já Lee et al. (1979 apud Lim et al., 2003) reportaram, para a mesma propriedade, o valor médio de $66 \mathrm{MPa}$. Quanto à rigidez, Lee et al. (1979 apud Lim et al., 2003) reportaram o valor médio de 9.240 MPa para a madeira de seringueira.

Analisando-se a face inferior (tracionada) dos corpos de prova ensaiados à flexão, 86,8\% dos exemplares apresentaram ruptura caracterizada como quebradiça (brash tension) e 13,2\% apresentaram ruptura caracterizada como desfibramento (splintering tension). Analisando-se a face lateral dos corpos de prova, $82,4 \%$ dos exemplares apresentaram ruptura caracterizada como simples (simple tension) e 17,6\% das rupturas foram caracterizadas como de tração com grã espiralada (cross-grain tension).

A dureza Janka, corrigida para a umidade padrão de $12 \%$ e transformada de megapascal $(\mathrm{MPa})$ para Newton $(\mathrm{N})$, variou de 5.728 a $6.706 \mathrm{~N}$ com coeficiente de variação de cada posição radial variando de 8,03 a 14,84\%. A análise de variância ( $\mathrm{p}$ valor $=0,022)$ mostrou que radialmente há variação estatisticamente significativa dessa propriedade, sendo verificado aumento do valor no sentido medula-casca. Esse comportamento é normalmente associado à juvenilidade do lenho das árvores. Os resultados são superiores aos reportados por Santana et al. (2001) - dureza Janka variando de 2.120 a $5.370 \mathrm{~N}$ - para a madeira de outros clones de seringueira a $12 \%$ de umidade e por Lee et al. (1979 apud Lim et al., 2003) - dureza Janka média de $3.030 \mathrm{~N}$ para madeira de seringueira úmida e $4.320 \mathrm{~N}$ para a madeira seca ao ar.

\section{CONCLUSÕES}

Com base nos resultados obtidos neste trabalho, podem-se apresentar as seguintes conclusões:

- A madeira do clone GT 1 de seringueira pode ser enquadrada na classe de resistência de dicotiledôneas $\mathrm{C} 40$, por apresentar resistência característica à compressão paralela às fibras maior que $40 \mathrm{MPa}$.

- No geral, não houve diferença estatisticamente significativa da variação das propriedades mecânicas analisadas na direção radial, porém houve tendência de aumento no valor dessas propriedades no sentido medula-casca.

- Não houve grande influência da juvenilidade nas propriedades mecânicas, com exceção da dureza Janka, que apresentou como comportamento o aumento de seus valores no sentido medula-casca.

\section{STATUS DA SUBMISSÃO}

Recebido: 30/08/2011

Aceito: 07/02/2012

Resumo publicado online: 22/03/2012

Artigo completo publicado: 30/06/2012

\section{AUTOR(ES) PARA CORRESPONDÊNCIA}

\section{Adriano Wagner Ballarin}

Departamento de Engenharia Rural, Faculdade de Ciências Agronômicas - FCA, Universidade Estadual Paulista - UNESP, Fazenda Experimental Lageado, Rua Doutor José Barbosa de Barros, 1780, CEP 18610-307, Botucatu, SP, Brasil

e-mail: awballarin@fca.unesp.br

\section{APOIO FINANCEIRO}

Fundação de Amparo à Pesquisa do Estado de São Paulo (FAPESP)

\section{REFERÊNCIAS}

American Society for Testing and Materials - ASTM. D143-94: standard test methods for small clear specimens of timber. West Conshohocken: ASTM; 2007.

Associação Brasileira de Normas Técnicas - ABNT. NBR 7190: Projeto de estruturas de madeira. Rio de Janeiro: ABNT; 1997.

Balsiger J, Bahdon J, Whiteman A. The utilization, processing and demand for rubberwood as a source of wood suplly. Rome: FAO; 2000.

Benjamin CA. Estudo da estrutura anatômica $e$ das propriedades físicas e mecânicas da madeira de Corymbia (Eucalyptus) citriodora e Eucalyptus grandis [tese]. Botucatu: Faculdade de Ciências Agronômicas, Universidade Estadual Paulista; 2006.

Bodig J, Jayne BA. Mechanics of wood and wood composities. 2nd ed. Malabar: Krieger; 1993. 
Colloquium on Rubberwood: resources and technologies; 1998; Kuala Lumpur. Kuala Lumpur: FRIM; 1998.

Eufrade Junior HJ, Ballarin AW, Leonello EC. Caracterização mecânica da madeira do clone RRIM 600 de seringueira (Hevea brasiliensis (Willd. ex. A. Juss.) Müll.Arg.) à compressão paralela às fibras. In: Anais do XXIII Congresso de Iniciação Científica; 2011; Botucatu. Botucatu: UNESP; 2011.

Hong LT. Rubberwood utilization: a success story. In: Proceedings of XX World Congress of the International Union of Forestry Research Organizations; 1996; Tampere. Tampere; 1995. [cited 2011 ago. 20]. Available from: http://www.metla.fi/iufro/iufro95abs/rsp19.htm.

Instituto Agronômico de Campinas - IAC. A importância da borracha natural. Programa Seringueira; 2009. [cited 2010 jun. 22]. Available from: http://www.iac. sp.gov.br/UniPesquisa/Seringueira/QuemSomos/ ImportanciaBorracha.asp.

Instituto Agronômico do Paraná - IAPAR. O Cultivo da seringueira (Hevea spp.). Secretaria de Estado da Agricultura e do Abastecimento; 2004. [cited 2009 out. 24]. Available from: http://www.iapar.br/arquivos/ File/zip_pdf/cultsering.pdf.

Lim SC, Gan KS, Choo KT. The characteristics, properties and uses of plantation timbers - rubberwood and Acacia mangium. Timber Technology Bulletin 2003; 26: 1-11.

Lorenzi H. Árvores brasileiras: manual de identificação e cultivo de plantas arbóreas nativas do Brasil. 3rd ed. Nova Odessa: Plantarum; 2000.

Matan N, Kyokong B. Effect of moisture content on some physical and mechanical properties of juvenile rubberwood (Hevea brasiliensis Muell. Arg.). Songklanakarin Journal of Science and Technology 2003; 25(3): 327-340.

May A, Gonçalves PS. Produtos complementares na exploração do seringal - matéria tecnica. Borracha Atual 1999; 17-21. [cited 2011 fev. 14]. Available from: http://www.borrachaatual.com.br/materiatecnica/20/ materia_tecnica_20a.pdf.
Okino EYA, Souza MR, Santana MAE, Sousa ME, Teixeira DE. Chapa aglomerada de cimentomadeira de Hevea brasiliensis Müll. Arg. Revista Árvore 2004; 28(3): 451-457.http://dx.doi.org/10.1590/ S0100-67622004000300016

Panshin AJ, Zeeuw C. Textbook of wood technology. 4th ed. New York: McGraw-Hill College; 1980.

Rocha RR. Avaliação não-destrutiva de madeiras para dormentes ferroviários [dissertação]. Botucatu: Faculdade de Ciências Agronômicas, Universidade Estadual Paulista; 2003.

Santana MAE, Eiras KMM, Pastore TCM. Avaliação da madeira de quatro clones de Hevea brasiliensis por meio de sua caracterização físico-mecânica. Brasil Florestal 2001; 70: 61-68.

Silva LL. Caracterização física e mecânica da madeira de seringueira (Hevea brasiliensis (Willd. ex. A. Juss.) Müll. Arg.) Botucatu: Faculdade de Ciências Agronômicas, Universidade Estadual Paulista; 2010. Relatório de Iniciação Científica.

Teixeira DE, Alves MVS, Costa AF, Sousa NG. Características de chapas de cimento-madeira com partículas de seringueira (Hevea brasiliensis Müell. Arg.) tratadas com CCA. Floresta $e$ Ambiente 2001; 8(1): 18-26.

Tomazello Filho M. Variação radial da densidade básica e da estrutura anatômica da madeira do Eucalyptus saligna e E. grandis. IPEF / Instituto de Pesquisas e Estudos Florestais 1985; 29: 37-45.

Tomazello Filho M. Variação da densidade básica em estrutura anatômica da madeira do Eucalyptus globulus, E. pellita e E. acmenioides. IPEF / Instituto de Pesquisas e Estudos Florestais 1987; 36: 35-42.

Zhao Y. Promotion of rubberwood processing technology in the Asia-Pacific region. In: Proceedings of ITTO/CFC International Rubberwood Workshop; 2008; Haikou. Haikou: ITTO; 2008. [cited 2010 mar. 15]. Available from: http://www.paneltech.cn/rubberwood/ WorkshoPresentations/WorkshopProceedings.pdf. 